\title{
Resonant x-ray scattering of magnetic anisotropy and orbital ordering in $\mathrm{Ca}_{2} \mathrm{RuO}_{4}$ \\ Dan Porter
}

\author{
Diamond Light Source Ltd, Didcot, United Kingdom; \\ dan.porter@diamond.ac.uk
}

$\mathrm{Ca}_{2} \mathrm{RuO}_{4}(\mathrm{CRO})$, the close neighbour of the famous superconductor $\mathrm{Sr}_{2} \mathrm{RuO}_{4}$ displays surprisingly different behaviour to its neighbour, exhibiting insulating behaviour below an irreversible metal-insulator transition at $\mathrm{T}_{\mathrm{MI}}=357 \mathrm{~K}$. In the insulating state $\mathrm{CRO}$ displays orbital ordering at $\mathrm{T}_{\mathrm{OO}}=260 \mathrm{~K}$ and antiferromagnetic ordering below $\mathrm{T}_{\mathrm{N}}=110 \mathrm{~K}$. This material has been extensively investigated but still questions remain regarding the nature of the insulating state and whether Mott gaps are opened only on certain orbitals, or whether the insulating state is a result of purely structural change. While recent publications have tended towards the latter of these possibilities, previous results observing varying orbital concentrations with temperature have not been explained. Here we will show new resonant elastic x-ray scattering (REXS) results from the Ruthenium absorption edge made on the synchrotron beamline I16 at Diamond. The resonant spectra provide a unique way of looking at the ordered magnetic and orbital structure of this material and we will present a systematic approach to understanding the different contributions to these signals.

Keywords: X-ray scattering, REXS, ruthenate, magnetism, orbital order 